\section{Who Pix up Rac1?}

embers of the large family of Rho GTPases are master regulators of the cytoskeletal changes necessary for cell adhesion, migration, and division. On page 759 , ten Klooster et al. show how the activity of a Rho GTPase is spatially restricted by its $\mathrm{COOH}$-terminal domain. The findings suggest that this highly variable region might provide the zip code for many different Rho members.

The $\mathrm{COOH}$-terminal domain is known for its lipid-anchoring ability. But since membranes abound in the cell, that ability did not explain the specific localization of different GTPases. A COOH-terminal peptide of Rac1 had been shown to disrupt normal Rac1 activity, so the authors used this domain to pull down interacting proteins. The screen picked up Rac1's own activator, an exchange factor called $\beta$-Pix.

$\beta$-Pix was found in focal adhesions at the leading edge of migrating cells, where Rac1 activity is known to drive polarized actin polymerization. $\beta$-Pix was also necessary for Rac1-mediated membrane ruffling.

The Rac1 binding site on $\beta$-Pix can also be occupied by an effector kinase called Pak1. The authors show that Pak1 binding to $\beta$-Pix normally prevents Rac1 activation. But Pak1 phosphorylation (as occurs upon integrin ligation) releases it from $\beta$-Pix, thus freeing $\beta$ Pix to recruit and activate Rac1.

The targeting mechanisms of most other Rho GTPase have not been determined. Given their highly divergent sequences, the COOH-terminal domains probably mediate interactions with a variety of adaptors that might control the localization of individual Rho GTPase members. JCB

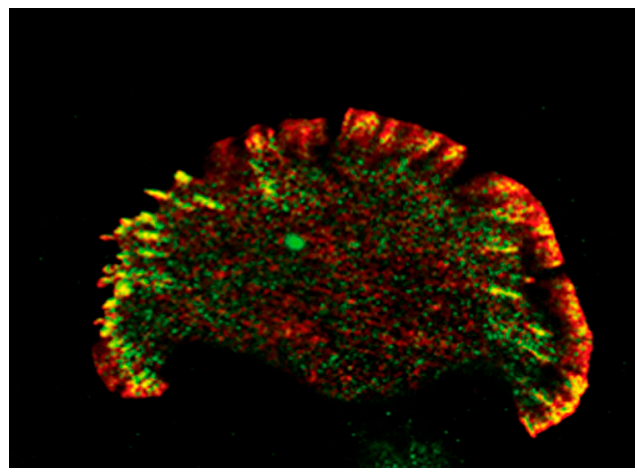

$\beta$-Pix (green) brings Racl (red) to ruffles and focal adhesions.
Par-3 for the cofilin n page 671, Chen and Macara unveil a mechanism by which Par-3 orchestrates cell polarization by regulating actin reorganization.

Par-3 is one member of an apical polarity-inducing complex that also includes Par-6 and aPKC. The Par proteins are well-known for protein-protein interactions, but how these interactions lead to polarization is less clear. Using RNAi, Chen and Macara had previously shown that Par-3 helps assemble tight junctions (TJs) in epithelial cells by inhibiting an activator of Rac. The study now reveals a second function of Par-3 at TJs.

In the previous work, the authors saw that TJ formation required actin reorganization, so Chen looked for any effects of Par-3 on actin regulators. He now finds that cofilin, an actin filament severing protein, is phosphorylated and thus inactivated in the absence of Par-3.

The inactivation of cofilin in the Par-3 RNAi cells is due to overactive LIMK2, which is one of several kinases that modifies cofilin. Par3 binds to LIMK2 and inhibits its kinase activity. By doing so at TJs, where the Par complex is localized, Par-3 would keep cofilin active, thus driving actin reorganization.

Par-3 and LIMK2 are widely expressed, so Chen suspects that actin might be regu-
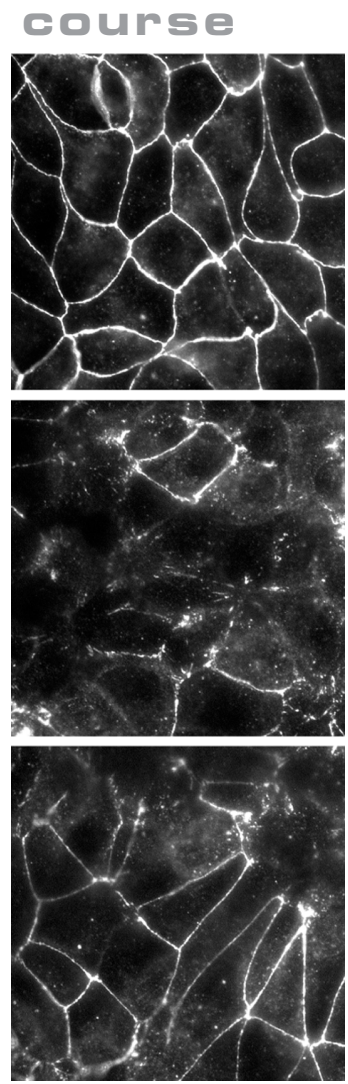

Tight junctions (top) disassemble without Par-3 (middle) but are rescued by active cofilin (bottom). lated by these proteins in other cell types. He is currently examining whether migrating cells require Par-3 to block LIMK2 at the leading edge, where actin turnover is needed. Јсв

\title{
Insulin pathway clears aggregates
}

he activation of part of a nutritional signaling pathway clears protein
aggregates, as shown by Yamamoto et al. (page 719). The findings sug-
gest that cells in crisis get an unusual outcome from insulin pathways.

Insulin signaling tells the cell that energy sources are present. It thus turns on protein translation and inactivates autophagy, which degrades bulk cytoplasmic proteins to supply starving cells with amino acids. The autophagy is turned on when insulin signaling activates an insulin receptor effector, called IRS-2, which then turns on a PI3K.

Yamamoto et al. found that aggregates of mutant huntingtin protein (htt) also turned on IRS-2, but that this increased autophagy. Insulin-activated and htt-activated IRS-2 had different activities because the latter activated a different PI3K, called hVps34, to clear htt aggregates via autophagy and lysosomal degradation. Thus, the insulin pathway, which is thought to convey a state of well-being, is now shown to help induce such a state.

Whether cytoplasmic aggregates are in fact toxic is still debated among scientists studying protein aggregation diseases, such as Huntington's and Parkinson's. If they are toxic, insulin might be useful to clear them up, but insulin administration in humans would have to be carefully controlled to avoid insulin shock. Direct activation of IRS-2/hVps34 might be a safer route. JCB 